\title{
Stochastic Modeling of the Expected Time to Search for an Intermittent Signal Source Under a Limited Sensing Range
}

\author{
Dezhen Song, Chang-Young Kim, and Jingang Yi
}

\begin{abstract}
A mobile robot is deployed to search for a stationary target that intermittently emits short duration signals. The searching mission is accomplished as soon as the robot receives a signal from the target. However, the robot cannot perceive the signal unless the target is within its limited sensing range. Therefore, the time to search the target is inherently random and hence unknown despite its importance in many searching and rescue applications. Here we propose the expected searching time (EST) as a metric to evaluate different robot motion plans under different robot configurations. We derive a closed form solution for computing the EST. To illustrate the EST model, we present two case studies. In the first case, we analyze two common motion plans: a slap method and a random walk. The EST analysis shows that the slap method is asymptotically faster than the random walk when the searching space size increases. In the second case, we compare a team of $n$ low-cost equallyconfigured robots with a super robot that has the sensing range equal to that of the summation of the $n$ robots. The EST analysis shows that the low-cost robot team takes $\Theta(1 / n)$ time and the super robot takes $\Theta(1 / \sqrt{n})$ time as $n \rightarrow \infty$. In both cases, our EST model successfully demonstrates its ability in assessing the searching performance. The analytical results are also confirmed in simulation.
\end{abstract}

\section{INTRODUCTION}

Mobile robots are often employed to perform searching tasks such as finding a black box in a remote area after an airplane crash, searching victims after an earthquake or a mine collapse disaster, or locating artifacts on the ocean floor. In many cases, the target can intermittently emit short duration signals to assist searching. For example, an airplane black box transmits radio signals periodically. An earthquake victim may knock the rubble from time to time. The searching task is accomplished once the robot detects the signal emitted by the target. However, the robot usually has a limited sensing range and cannot detect the target that is out of the range. It seems straightforward that we can use the traditional coveragebased motion plans to guide the robot to cyclically scan the searching space to locate the target. However, the time to search the target is inherently random and hence remains unknown despite its importance in many searching and rescue applications.

This work was supported in part by the National Science Foundation under CAREER grant IIS-0643298 and MRI-0923203.

D. Song and C. Kim are with CSE Department, Texas A\&M University, College Station, TX 77843, USA, (emails: dzsongecse.tamu.edu and kcyoung@cse.tamu.edu).

J. Yi is with MAE Department, Rutgers University, Piscataway, NJ 08854 USA, (email: jgyi@rutgers.edu).

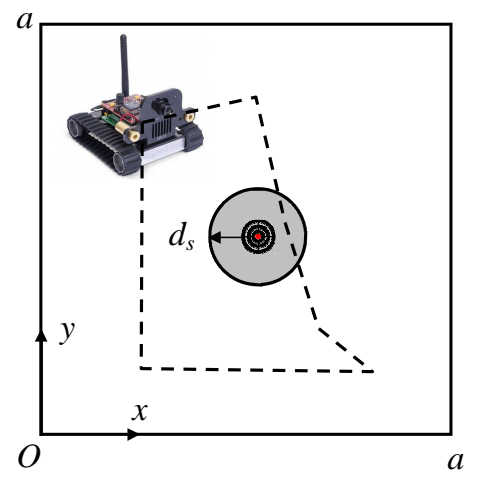

Fig. 1. A robot attemps to search for a target (the red dot) that intermittently emits short duration signals in a square. The gray circle is the region that the robot can sense the signal from the target. The dashed line is a robot trajectory.

To address this new problem, we propose the expected searching time (EST) as a metric for the searching ability. We model the searching process as a delayed alternating renewal process and derive the EST as a function of the searching space size, the signal transmission rate, and the robot sensing range. The resulting closed-form solution of the EST can be used to analyze the searching efficiency for different robot configurations and searching plans. Since the model components can be obtained from online measurements and known robot parameters, a great benefit of the resulting model is that it is capable of predicting the EST for an ongoing searching process. This characteristic is important for timecritical searching and rescue applications.

The contributions of the paper are trying to bring analytical results to interpret well-known searching strategies. The EST analysis not only can reveal our common believes about existing searching methods but also predict how the effectiveness of those methods changes as trajectory selection, sensing range, searching space size, and robot distribution change. Building on the latest development in random walk in constrained space from stochastic modeling community, our analysis for the first time show that traditional slap method $\left(\Theta\left(a^{2}\right)\right)$ actually is asymptotically faster than the random walk $\left(O\left(a^{2} \log a\right)\right)$ for a squared searching space of the size length $a$, which is never known before. In the second case, we compare a team of $n$ identically-configured low-cost robots with a super robot that has the sensing coverage equal to the summation of the $n$ lowcost robots. The EST analysis shows that the low-cost robot team outperforms the super robot because its EST is $\Theta(1 / n)$ while the EST for the super robot is $\Theta(1 / \sqrt{n})$ as $n \rightarrow \infty$. 
Again, this new analytical result has not been seen before and is important for developing new search strategies.

The analytical results are confirmed in simulation for both cases. The EST model successfully demonstrates its ability in assessing the searching performance under different robot configurations and motion plans.

The rest of the paper is organized as follows. We begin with the related work in Section II. We define the problem in Section III. We derive the EST in a closed form in Section IV. The two case studies are presented in Section V. The analytical results are validated in simulation in Section VI before we conclude the paper.

\section{RELATED WORK}

Searching an object in physical space is one of the most important tasks for robots or humans. When prior information such as the spatial distribution of the target is known, this is comparable to the foraging behavior of animal [1]. However prior target information is often not available. If the target is continuously emitting signals, just simply scanning the entire searching space once enables the robot to find the target. Since the worst case for the searching time is the time to cover the entire searching space, the searching problem becomes a coverage problem [2]-[4]. For a known environment, a coverage problem for a single robot often employs different approaches to decompose the searching space and output a continuous path that allows the robot to cover the entire searching space. If the searching space can be modeled as a set of $w$-disjoint discrete choices, searching a target with a limited sensing range and $w$-choice is known as a $w$-lane Cow-Path problem [5].

While the running time is well understood for the coverage problems [6], [7], this is not the case when the searching process depends on the signal emitted by the target because the collocation of the robot and the target does not necessarily mean that the target is found. When the target is not emitting a signal, the robot cannot find the target. The robot has to keep scanning the searching space. The deterministic coverage algorithm becomes a Las Vegas algorithm [8] where the target will eventually be found but the searching time is random. However, the searching time can be crucial for many searching tasks. For example, victims of an earthquake often have limited survival time. Although searching itself is a very old problem, few models exist for analyzing the effectiveness of a searching strategy when the source is intermittently emitting signals.

Another set of related work is robot exploration and mapping problems where the environment is not previously known [9]. The task is not only to cover the entire space but also to output the true representations of the environment. Recent advances in using a multi-robot team to perform exploration and mapping tasks mainly focuses on the coordination of the robot/sensor team [10]-[15] under various dynamics, communication, sensing, and energy constraints. Although not directly applicable to our problem, researchers have accumulated interesting empirical results that are using a team of low-cost robots usually performs faster and more fault-tolerant [14] than a single expensive robot. This really inspires our problem because we want to see if our analytical model can show similar results under similar constraints/conditions.

Our group has built experience in searching for targets that intermittently transmit signals by developing algorithms and systems to detect an unknown wireless sensor network [16][18]. In these problems, the robot can accumulate the information about the target location over time through the signal strength readings and antenna models. The searching problem is less difficult because the robot can utilize the information in the planning process. However, such information is often not available in many searching tasks, which is the focus of this paper.

\section{PRoblem Definition}

As illustrated in Fig. 1, a single robot searches for a single target in a squared 2D Euclidean space with a side length of $a$. Define $d_{s}$ as the maximum sensing distance of the sensor on the robot. The robot travels at the constant speed of $v$. To formulate the problem and focus on the most relevant issues, we make the following assumptions,

1) There is no prior information about possible locations of the target. Therefore, the target is assumed to be uniformly distributed in the searching space. This is actually the most difficult searching case.

2) The target transmits short duration signals periodically according to a Poisson process with a known rate $\lambda$. The signal duration is short due to energy concerns. A Poisson process is a good approximation to a general random arrival process in stochastic modeling [19]. In some cases, the target may be a continuous beacon; but it is very difficult to be detected due to environment conditions or unreliable sensing, which can also be modeled as a target with intermittent signals.

3) During the searching process, either the target is static or its movements are negligible in comparison to $d_{s}$. The searching space is much larger than the sensing distance: $a \gg d_{s}$.

Condition 1 (Sensing Condition): The robot cannot sense the signal unless an actively-transmitting target is within distance $d_{s}$ due to the sensing range limit.

As illustrated in Fig. 1, this defines a circle centered at the target with the radius of $d_{s}$, which is the region that robot has a chance to sense the target. We refer to the region as "the circle" in the rest of the paper. Due to the fact that the robot does not know the location of the target, the actual position of the circle in the searching space is also unknown.

Condition 2 (Termination Condition): The searching task is accomplished as soon as the robot senses a signal.

Condition 2 implies that the robot cannot find an inactive target even it is collocated with the target. For example, an airplane is not be able to notice the survivor on an island if the person does not send a signal (e.g. fire or smoke). On the other hand, only one signal reception is needed in the searching process. Conditions 1 and 2 establish a new type of searching problem as oppose to a regular coverage problem. Let us define $T_{s}$ as the searching time for the robot to find the target. Therefore, our problem is defined as follows, 
Problem 1 (The EST Computation): Given $\lambda, d_{s}$, and $a$, calculate the EST $E\left(T_{s}\right)$, where $E(\cdot)$ denotes the expected value function.

\section{MOdELING}

One immediate question about Problem 1 is whether we can obtain the EST without referring to or being limited to a particular motion plan. To address this dependency, we first characterize the motion plans based on their outcomes before modeling the EST.

\section{A. Characterizing Planners}

Periodically, the robot planner outputs a motion plan and the system is naturally a repetitive scanning process. We name its trajectory in each period as a tour. The dashed line in Fig. 1 illustrates a tour.

Definition 1: A tour starts at the moment when the robot enters the target circle and ends at next moment when the robot enters the circle again.

Tours may be quite different based on the planner. For example, tour length varies each time if the robot follows a random walk. As another example, a deterministic planner usually has a fixed tour trajectory.

Based on Condition 2, we know that the robot does not accumulate the knowledge regarding the target location from tours to tours because no signal has been perceived before the moment the searching mission is accomplished. Hence we can treat each tour as independently and identically distributed (i.i.d.). This allows us to model the searching process as a renewal process.

When a tour begins, the robot first spends some time inside the circle, which is defined as $\tau_{\mathrm{IN}}$. After that, the robot leaves the circle and spends some time before entering the circle again, where the next tour starts. This yields an alternating renewal process. The duration outside the circle is defined as $\tau_{\text {oUT }}$. Hence $\tau_{\text {IN }}+\tau_{\text {oUT }}$ is the overall duration for the tour.

\section{B. Modeling the EST}

Without loss of generality, we assume the robot starts the searching process from the origin which is on the boundaries of the searching space. It takes some time to reach the circle where the first tour starts. Define the time as delay $D$. From Conditions 1 and 2, we know that the robot cannot find the target in $D$. The searching process is a delayed alternating renewal process. Define $T_{s}^{c}$ as the time to find the target after the robot enters the repetitive tours. Hence, the EST is

$$
E\left(T_{s}\right)=E(D)+E\left(T_{s}^{c}\right) .
$$

Define $N$ as the number of signal transmissions during $\tau_{\text {IN }}$ in a tour. Since the arrival process of the signal transmission is Poisson, $N$ conforms to a Poisson distribution,

$$
P(N=k)=\frac{e^{-\lambda \tau_{\mathrm{IN}}}\left(\lambda \tau_{\mathrm{IN}}\right)^{k}}{k !}, \quad k=0,1,2, \ldots, \infty .
$$

We know that event $N>0$ means that at least one signal transmission happens during $\tau_{\mathrm{IN}}$. This means the target is found. Therefore, we can compute $E\left(T_{s}^{c}\right)$ by conditioning on $N$,

$$
E\left(T_{s}^{c}\right)=E\left(T_{s}^{c} \mid N>0\right) P(N>0)+E\left(T_{s}^{c} \mid N=0\right) P(N=0),
$$

where $P(N>0)=1-e^{-\lambda \tau_{\mathrm{IN}}}$ and $P(N=0)=e^{-\lambda \tau_{\mathrm{IN}}}$ according to (2).

Now let us compute $E\left(T_{s}^{c} \mid N>0\right)$. Since event $N>0$ is equivalent to event $T_{s}^{c} \leq \tau_{\mathrm{IN}}$, we have

$$
\begin{aligned}
E\left(T_{s}^{c} \mid N>0\right) & =E\left(T_{s}^{c} \mid T_{s}^{c} \leq \tau_{\mathrm{IN}}\right) \\
& =\frac{1}{\lambda}-\frac{\tau_{\mathrm{IN}} e^{-\lambda \tau_{\mathrm{IN}}}}{1-e^{-\lambda \tau_{\mathrm{IN}}}}
\end{aligned}
$$

because the conditional distribution $T_{s}^{c} \mid T_{s}^{c} \leq \tau_{\text {IN }}$ is a truncated exponential distribution. It is worth noting that (4) is valid only if $\tau_{\text {IN }}>0$. This is guaranteed according to Definition 1. On the other hand, we know

$$
E\left(T_{s}^{c} \mid N=0\right)=\tau_{\mathrm{IN}}+\tau_{\text {ouT }}+E\left(T_{s}^{c}\right)
$$

because the robot cannot find the inactive target in the current tour and has to start all over again in next tour.

Plugging (4) and (5) into (3) and (1), we have the following Theorem.

Theorem 1: Given the expected time $E(D)$ for the robot to reach the circle, the Poisson arrival rate of signal $\lambda$ of the target, the traveling time $\tau_{\mathrm{IN}}$ inside the circle, and the traveling time $\tau_{\text {out }}$ outside the circle, the EST of the target is

$$
E\left(T_{s}\right)=E(D)+\frac{1}{\lambda}+\tau_{\text {out }} \frac{e^{-\lambda \tau_{\mathrm{IN}}}}{1-e^{-\lambda \tau_{\mathrm{IN}}}} .
$$

Theorem 1 has a surprisingly succinct format revealing the relationship between the EST and the corresponding variables. To further explain (6), let us consider the following extreme cases:

Case 1: When $\lambda \rightarrow \infty$, it means that the target continuously transmits signals. An example is that a lost hiker keeps fire burning. Hence the light and the smoke of the fire become the continuous signal. Now the searching time becomes the time that it takes for the robot to enter the circle. The problem degenerates to the traditional coverage problem where $E\left(T_{s}\right)=E(D)$.

Case 2: When $\tau_{\text {out }}=0$, it means that the signal emitted by the target is so powerful that the circle defined by $d_{s}$ can cover the entire searching space. In this case, $E(D)=0$. Hence $E\left(T_{s}\right)=1 / \lambda$. This is sensible because the result means the robot can find the target as soon as it emits a signal.

Case 3: When $\tau_{\text {IN }} \rightarrow 0$, which happens when $d_{s}$ is infinitesimally small, we have $E\left(T_{s}\right) \rightarrow \infty$. This result conforms to our expectation.

Remark 1: It is worth noting that (6) does not depend on a particular motion plan or the shape/dimension of the searching space, which makes it widely applicable in practice. Actually, the EST can be also applied to analyze searching tasks carried by humans. In many cases, the signal transmission rate $\lambda$ is known; $E(D)$ can be estimated based on observations; $\tau_{\text {IN }}$ can be estimated based on $d_{s}$ and $v$; and $\tau_{\text {OUT }}$ can be measured based on observations that how often a robot would revisit a region with the same size of the circle. Based on the known 
information and online measurements, we can even predict the EST for an ongoing searching process regardless its motion plan, which is of great importance in applications where the searching time literally means life or death.

\section{Analysis of Common Searching Strategies}

Theorem 1 can be used to analyze the searching performance under different robot motion plans and configurations. We begin with demonstrating how Theorem 1 can reveal the difference between two motion plans from common coverage methods, namely, the slap method and the random walk.

\section{A. The Slap Method}

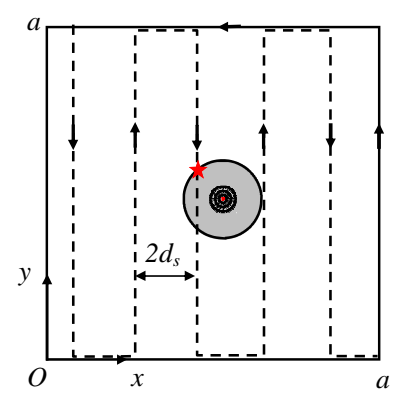

(a)

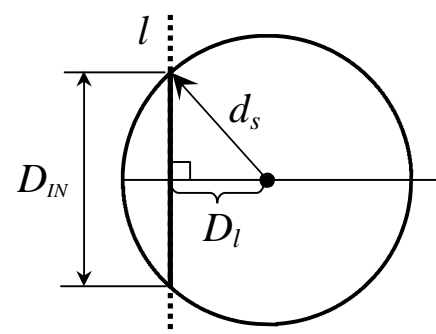

(b)
Fig. 2. (a) A sample motion plan for the slap method. (b) An illustration of how a tour (line $l$ ) intersects the circle of the target.

The slap method [20], also known as the trapezoidal decomposition [21] in robotics, sequentially scans the entire searching space back and forth. Fig. 2(a) gives the robot motion plan for the square case. The plan is a set of $y$ axis parallel lines (appears to be vertical lines in Fig. 2(a)) that cover the entire searching space. The vertical lines are inter-connected using the boundaries of the searching space to formulate a complete tour. To guarantee an intersection between the circle and the tour, the distance between adjacent vertical lines is set to be $2 d_{s}$.

The red " $\star$ " in Fig. 2(a) is the starting point of the tour. Since tours are exactly the same in the slap method, the subsequent tours start exactly at the same location. The overall tour length is approximately $a^{2} /\left(2 d_{s}\right)$. Given the robot speed $v$, we know it takes

$$
\tau_{\mathrm{IN}}+\tau_{\mathrm{OUT}} \approx \frac{a^{2}}{2 v d_{s}}
$$

time for the robot to finish the tour. Since the target could be anywhere in the searching space with equal probabilities, we know that

$$
E(D) \approx\left(\tau_{\text {IN }}+\tau_{\text {ouT }}\right) / 2=\frac{a^{2}}{4 v d_{s}} .
$$

The remaining undetermined variable is $\tau_{\mathrm{IN}}$. Let us define $D_{\text {IN }}$ as the distance traveled inside the circle. $D_{\text {IN }}$ is the length of intersection when the line intersects the circle as illustrated in Fig. 2(b). Here we ignore the boundary effect where the circle is not a full circle because $a \gg d_{s}$. Line $l$ in Fig. 2(b) is a part of the tour. When $l$ intersects the circle, we define
$D_{l}$ as the distance between the center of the circle and the line. Since the target is uniformly distributed in the 2D space, $D_{l} \sim U\left(0, d_{s}\right)$ is uniformly distributed. From Fig. 2(b), we know

$$
\tau_{\mathrm{IN}}=\frac{D_{\mathrm{IN}}}{v}=\frac{2 \sqrt{d_{s}^{2}-D_{l}^{2}}}{v} .
$$

Plugging (7), (8) and (9) into (6) and conditioning on $D_{l}$, we have,

$$
E\left(T_{s} \mid D_{l}\right) \approx \frac{a^{2}}{4 v d_{s}}+\frac{1}{\lambda}+\left(\frac{a^{2}}{2 v d_{s}}-\frac{2 \sqrt{d_{s}^{2}-D_{l}^{2}}}{v}\right) \phi\left(\lambda, D_{l}\right)
$$

where

$$
\phi\left(\lambda, D_{l}\right)=\frac{1}{e^{\frac{2 \lambda \sqrt{d_{s}^{2}-D_{l}^{2}}}{v}}-1} .
$$

Since $a \gg d_{s}, \tau_{\text {OUT }} \gg \tau_{\text {IN }}$, and $\frac{2 \sqrt{d_{s}^{2}-D_{l}^{2}}}{v}$ is negligible if compared with $\frac{a^{2}}{2 v d_{s}}$, we have,

$$
E\left(T_{s} \mid D_{l}\right) \approx \frac{a^{2}}{4 v d_{s}}+\frac{1}{\lambda}+\frac{a^{2}}{2 v d_{s}} \phi\left(\lambda, D_{l}\right)
$$

Hence we have the EST for the slap method,

$$
\begin{aligned}
E\left(T_{s}\right) & =\int_{\delta=0}^{d_{s}} E\left(T_{s} \mid D_{l}=\delta\right) \frac{1}{d_{s}} d \delta \\
& \approx \frac{a^{2}}{4 v d_{s}}+\frac{1}{\lambda}+\frac{a^{2}}{2 v d_{s}} g\left(d_{s}, \lambda\right)
\end{aligned}
$$

where

$$
g\left(d_{s}, \lambda\right)=E\left(\phi\left(\lambda, D_{l}\right)\right)=\int_{\delta=0}^{d_{s}} \frac{1}{d_{s}} \phi\left(\lambda, D_{l}\right) d \delta .
$$

Let $\delta=d_{s} \cos \theta$, we can transform (14) into

$$
g\left(d_{s}, \lambda\right)=\int_{\theta=0}^{\pi / 2} \frac{1}{e^{\frac{2 \lambda d_{s} \sin \theta}{v}}-1} \sin \theta d \theta .
$$

When $\lambda$ and $d_{s} / v$ are very small, (14) can be further simplified,

$$
g\left(d_{s}, \lambda\right) \approx \frac{\pi v}{4 \lambda d_{s}}-1
$$

Remark 2: Eq. (13) also suggests that a fast robot (large $v$ ) with great sensing distance $d_{s}$ reduces the EST. This conclusion agrees with our intuition that mobility and sensing are the key elements in searching. However, it also takes a target's cooperation to further reduce the EST. When the robot reaches its speed and sensing limit, the only way to reduce EST is to increase $\lambda$. Of course, the target usually has energy constraints and cannot arbitrarily increase $\lambda$.

The analysis assumes the distance between vertical lines is $2 d_{s}$, which ensures there is only one intersection between the circle and the tour. When a smaller spacing is used, the overall tour length increases and so does $\tau_{\mathrm{IN}}$. The analysis is slightly more complicated because it needs to be conditioned on the number of intersections between the tour and the circle. The results actually share a similar format with (15) and the same asymptotical properties with respect to $a, v$, and $\lambda$. Since our focus is to compare the asymptotic behavior of the slap method with that of the random walk, we omit the analysis here.

Another reasonable concern is that whether ignoring the 
boundary effect impacts the final result. When the circle is located at the boundary of the square, distance $D_{\text {IN }}$ cannot be computed using (9). Since the target has to be located within $d_{s}$ distance of the boundary to create the scenario, the probability that such event happens is less than $\frac{4 d_{s} a}{a^{2}}=\frac{4 d_{s}}{a} \ll 1$, since $d_{s} \ll a$. Hence its impact to the final EST is ignorable because $D_{\mathrm{IN}}$ for such case is not significantly different from that of the non-boundary case. Therefore, we will ignore boundary effect in the rest of the paper.

\section{B. Random Walk}

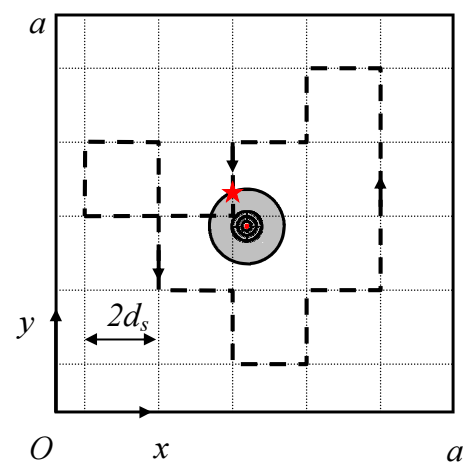

Fig. 3. An illustration of robot motion plan based on a 2D lattice-based random walk

Another popular motion plan is to employ a 2D random walk to traverse the searching space. As illustrated in Fig. 3, we partition the entire searching space using a $2 \mathrm{D}$ finite lattice with a spacing of $2 d_{s}$ in each dimension. Denoting $N_{s}$ as the number of lattice nodes, we have $N_{s}=\frac{a^{2}}{4 d_{s}^{2}}$ nodes. Finer lattice is possible but usually associated with higher energy cost because the robot has to make a lot more turns.

The robot always moves from one lattice node to its neighboring node with equal probabilities. The robot does not cross the boundaries. According to [22], this is a finite 2D lattice with reflective boundaries. Recall that a tour starts at the moment the robot enters the circle. Since the robot might not enter the circle at the exactly same location in different tours, each tour is not necessarily a completely closed curve as that in the slap method case. The closed curve tour in Fig. 3 only happens with a probability of $1 / 4$.

To compute the EST in (6), we need to compute $E(D)$. Recall that the robot always starts at origin. Given the location of target $\left(X_{t}, Y_{t}\right)$, computing the mean time that it takes the robot to follow the random walk to reach a particular location $\left(X_{t}, Y_{t}\right)$ is the mean first passage time (MFPT) [23], [24] problem in stochastic modeling. The exact solution to this problem is expressed in the format of pseudo Green functions and cannot be explicitly analyzed. Since $a \gg d_{s}$, there are a large number of nodes $\frac{a^{2}}{4 d_{s}^{2}}$ in the 2D lattice and each robot move takes $\frac{2 d_{s}}{v}$ time. Hence we can apply the recent results of MFPT using its asymptotic format in [25],

$$
E\left(D \mid X_{t}, Y_{t}\right) \approx \frac{a^{2}}{2 v d_{s}}\left(\alpha_{0}+\alpha_{1} \ln \sqrt{X_{t}^{2}+Y_{t}^{2}}\right),
$$

where $\alpha_{0}$ and $\alpha_{1}$ are constants and can be determined by Monte Carlo methods. According to [25], $\alpha_{0}$ and $\alpha_{1}$ strikingly do not depend on lattice size but local transitional properties. Hence,

$$
\begin{aligned}
E(D) & =\int_{0}^{a} \int_{0}^{a} E\left(D \mid X_{t}=x, Y_{t}=y\right) \frac{1}{a^{2}} d x d y \\
& \approx \frac{\alpha_{0} a^{2}}{2 v d_{s}}+\frac{\alpha_{1}}{2 v d_{s}} \int_{0}^{a} \int_{0}^{a} \ln \sqrt{x^{2}+y^{2}} d x d y .
\end{aligned}
$$

Since

$$
\int_{0}^{a} \int_{0}^{a} \ln \sqrt{x^{2}+y^{2}} d x d y=a^{2} \ln a+\frac{\pi+2 \ln 2-6}{4} a^{2}
$$

we have

$$
E(D) \approx \frac{a^{2}}{2 v d_{s}}\left(\alpha_{0}+\alpha_{1} \ln a+\alpha_{1} \frac{\pi+2 \ln 2-6}{4}\right) .
$$

The remaining unknown term in (6) is $E\left(\tau_{\text {OUT }} \frac{e^{-\lambda \tau_{\mathrm{IN}}}}{1-e^{-\lambda \tau_{\mathrm{IN}}}}\right)$. Given the robot speed $v, \tau_{\mathrm{IN}}$ is uniquely determined by the distance in the circle $D_{\text {IN }}$, which is independent of the overall trajectory. Also $E\left(\tau_{\text {out }}\right) \approx E\left(\tau_{\text {ouT }}+\tau_{\text {IN }}\right)$ given that $a \gg d_{s}$. Hence,

$$
E\left(\tau_{\text {OUT }} \frac{e^{-\lambda \tau_{\mathrm{IN}}}}{1-e^{-\lambda \tau_{\mathrm{IN}}}}\right) \approx E\left(\tau_{\text {OUT }}+\tau_{\mathrm{IN}}\right) E\left(\frac{e^{-\lambda \tau_{\mathrm{IN}}}}{1-e^{-\lambda \tau_{\mathrm{IN}}}}\right) .
$$

Since the 2D lattice-based random walk is undirected and symmetric in transitional probability, we know that the stationary probability of staying inside the circle is $p_{c}=\frac{\pi d_{s}^{2}}{a^{2}}$. Therefore, we know the following is true according to Renewal Reward theorem,

$$
\frac{E\left(\tau_{\text {IN }}\right)}{E\left(\tau_{\text {OUT }}+\tau_{\text {IN }}\right)}=p_{c}=\frac{\pi d_{s}^{2}}{a^{2}} .
$$

Plugging (22) into (21), we have

$$
E\left(\tau_{\text {ouT }} \frac{e^{-\lambda \tau_{\mathrm{IN}}}}{1-e^{-\lambda \tau_{\mathrm{IN}}}}\right) \approx \frac{a^{2}}{\pi d_{s}^{2}} E\left(\tau_{\mathrm{IN}}\right) E\left(\frac{e^{-\lambda \tau_{\mathrm{IN}}}}{1-e^{-\lambda \tau_{\mathrm{IN}}}}\right) .
$$

Now, we focus on the computation of $\tau_{\text {IN }}$. Since the lattice has a spacing of $2 d_{s}$, two scenarios exist when the tour on the lattice intersects the circle: i) the nearest lattice point on the tour is inside the circle and ii) the nearest lattice point on the tour is outside the circle as illustrated in Fig. 4. Let us define events that scenarios i) and ii) happen as events $E_{i}$ and $E_{o}$, respectively. Since the circle center is uniformly located in the searching space,

$$
P\left(E_{i}\right)=\frac{\pi d_{s}^{2}}{4 d_{s}^{2}}=\frac{\pi}{4}=1-P\left(E_{o}\right) .
$$

When event $E_{o}$ happens, we know that the robot trajectory intersects the circle as a straight line as shown in Fig. 4(a). Hence we have

$$
\tau_{\mathrm{IN}} \mid E_{o}=\frac{D_{\mathrm{IN}}}{v},
$$

where $D_{\text {IN }}$ is defined in (9) and the right side of $\mid$ is the condition for the equality to be true. This is a notation convention widely used in stochastic modeling [19]. Hence

$$
\begin{aligned}
E\left(\tau_{\mathrm{IN}} \mid E_{o}\right) & =\frac{\pi d_{s}}{2 v}, \text { and } \\
E\left(\frac{e^{-\lambda \tau_{\mathrm{IN}}}}{1-e^{-\lambda \tau_{\mathrm{IN}}}} \mid E_{o}\right) & =g\left(d_{s}, \lambda\right) .
\end{aligned}
$$




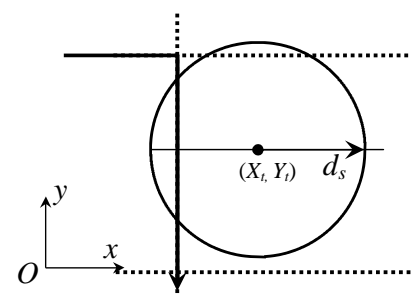

(a)

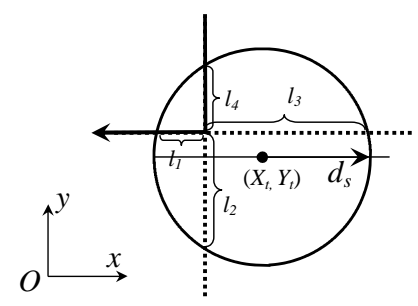

(b)
Fig. 4. An illustration of how the robot trajectory in solid line intersects the circle. (a) Scenario i): when the nearest lattice point on tour is located outside the circle. (b) Scenario ii): when the nearest lattice point on tour is located inside the circle. The dashed line in the figure is part of the lattice.

When event $E_{i}$ happens, one lattice point is inside the circle. As illustrated in Fig. 4(b), the lattice point inside the circle partitions the lattice edges inside the circle into four parts: $l_{1}, l_{2}, l_{3}$, and $l_{4}$. When a robot trajectory intersects the circle, the part of the trajectory inside the circle can be divided into two segments, which are defined as $L^{\prime}$ and $L^{\prime \prime}$, respectively. $L^{\prime}$ refers to the segment that the robot takes to arrive at the lattice node and $L^{\prime \prime}$ refers to the segment that the robot takes to leave the circle. Hence

$$
\tau_{\mathrm{IN}} \mid E_{i}=\frac{L^{\prime}+L^{\prime \prime}}{v} .
$$

Since $L^{\prime}$ and $L^{\prime \prime}$ have equal probabilities to take $l_{1}, l_{2}, l_{3}$, and $l_{4}$, there is a total of 16 combinations. Conditioning on the 16 $\left(L^{\prime}, L^{\prime \prime}\right)$ combinations and the circle center location $\left(X_{t}, Y_{t}\right)$, we get the same results as shown in (26) and (27). Combining those results for the $E_{i}$ and $E_{o}$ events by conditioning on them, we have the unconditional expected values,

$$
\begin{aligned}
E\left(\tau_{\mathrm{IN}}\right) & =\frac{\pi d_{s}}{2 v}, \text { and } \\
E\left(\frac{e^{-\lambda \tau_{\mathrm{IN}}}}{1-e^{-\lambda \tau_{\mathrm{IN}}}}\right) & =g\left(d_{s}, \lambda\right),
\end{aligned}
$$

where $g(\cdot)$ is defined in (15). Plugging (20), (23), (28), and (29) into (6), we can obtain the EST for the random walk case,

$$
\begin{aligned}
E\left(T_{s}\right) \approx & \frac{a^{2}}{2 v d_{s}}\left(\alpha_{0}+\alpha_{1} \ln a+\alpha_{1} \frac{\pi+2 \ln 2-6}{4}\right) \\
& +\frac{1}{\lambda}+\frac{a^{2}}{2 d_{s} v} g\left(d_{s}, \lambda\right) .
\end{aligned}
$$

Comparing (30) to (13), we have the follow conclusion,

Corollary 1: With the same field side length $a$, the sensing range $d_{s}$, and the signal transmission rate $\lambda$, the $E\left(T_{s}\right)$ value of the slap method is asymptotically smaller than that of the random walk when $a \rightarrow \infty$.

Proof: It is straightforward because $E\left(T_{s}\right)=\Theta\left(a^{2}\right)$ for the slap method from (13) while $E\left(T_{s}\right)=\Theta\left(a^{2} \ln a\right)$ for the random walk according to (30).

\section{Analysis of Different Robot Configurations}

Theorem 1 can also be used to analyze cases under different robot configurations. Here we compare two configurations.

A low-cost robot team (LCRT) case: We have $n$ identicallyconfigured low-cost robots. To coordinate the searching, we partition the searching space into $n$ sub square fields with an area of $a^{2} / n$ each and allocate one robot for each sub square field.

A single expensive robot (ASER) case: We have an expensive robot equipped with a very capable sensor that has a sensing area equal to the combination of those of the $n$ lowcost robots. If each of the low-cost robot has a sensing range of $d_{s}$, then the area of the combined sensing region for $n$ robots is $n \pi d_{s}^{2}$. Therefore, the sensing distance for the expensive robot is set to $d_{s}^{\prime}=\sqrt{n} d_{s}$ to ensure the same-sized sensing coverage at any given time.

We are now ready to compare these two robot configurations. Since the slap method is asymptotically faster than the random walk, we build on the slap method results in (30). For the LCRT, only one robot actually has the target in its sub field. Hence, the rest of $n-1$ robots are irrelevant in the searching process. Comparing with the original EST in (30), we just need to replace $a$ with $\frac{a}{\sqrt{n}}$. Defining the searching time for the LCRT as $T_{s}^{\prime}$, we have

$$
E\left(T_{s}^{\prime}\right) \approx \frac{a^{2}}{4 v n d_{s}}+\frac{1}{\lambda}+\frac{a^{2}}{2 v n d_{s}} g\left(d_{s}, \lambda\right) .
$$

Defining the searching time for the ASER as $T_{s}^{\prime \prime}$, we have

$$
E\left(T_{s}^{\prime \prime}\right) \approx \frac{a^{2}}{4 v \sqrt{n} d_{s}}+\frac{1}{\lambda}+\frac{a^{2}}{2 v \sqrt{n} d_{s}} g\left(\sqrt{n} d_{s}, \lambda\right) .
$$

From (15), it is not difficult to see that

$$
g\left(\sqrt{n} d_{s}, \lambda\right) \rightarrow 0 \text { as } n \rightarrow \infty .
$$

Therefore, we have the following conclusion,

Corollary 2: When traveling at the same velocity $v$, the low-cost robot team can find the target asymptotically faster than the single expensive robot does when $n$ increases, if $1 / \lambda$ is not the dominating factor in the EST.

Proof: From (32) and (33), we know $E\left(T_{s}^{\prime \prime}\right)=\Theta\left(\frac{1}{\sqrt{n}}+\right.$ $\left.\frac{1}{\lambda}\right)$. From (31), we know $E\left(T_{s}^{\prime}\right)=\Theta\left(\frac{1}{n}+\frac{1}{\lambda}\right)$. Hence the conclusion follows.

It is actually rather surprising to see the result in Corollary 2 at the first sight. We have not expected such a significant difference in the comparison. This conclusion is rather interesting because it shows that an expensive robot with superior sensing capability is not as good as a large number of low-cost robots with less capable sensors when searching for targets that intermittently transmits short duration signals.

Remark 3: This analysis also shows that if there are cost functions associated with the number of robots, different sensor options, or different velocity options available, we can use the EST results as an objective function to optimize the robot configuration for the task.

\section{EXPERIMENTS}

We test our results using Monte Carlo simulation. The simulation program is written in Microsoft Visual C++.Net 2005 on a Desktop PC with an 32-bit Windows XP Professional Edition OS. The Desktop PC has an Intel 2.13 GHz Core2Duo CPU with 2GB RAM and a 250 GB Hard disk.

The experimental results are illustrated in Figs. 5 and 6. Each data point in both figures is an average of 10,000 


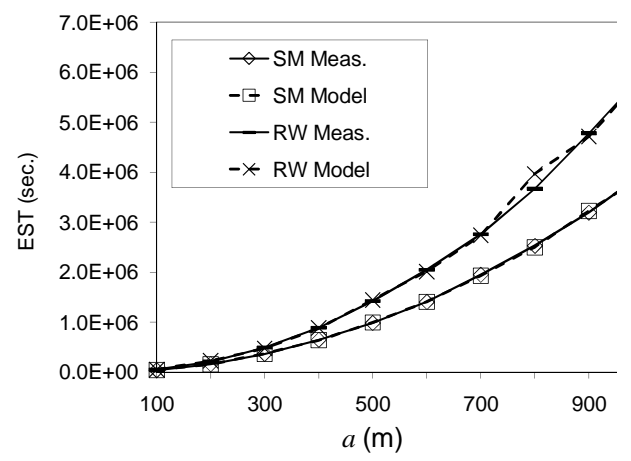

(a)

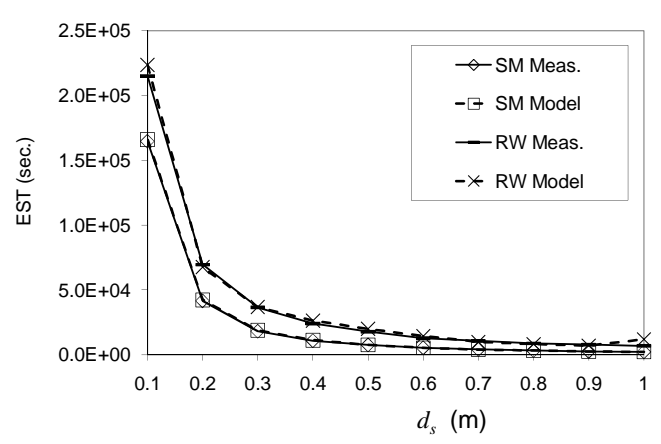

(c)

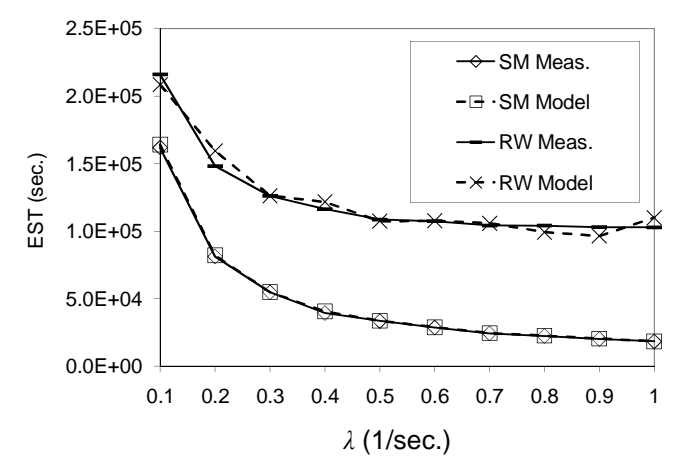

(b)

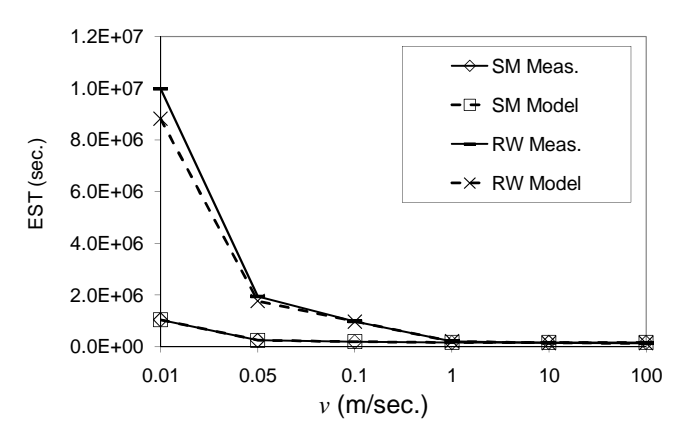

(d)

Fig. 5. Simulation results in (a), (b), (c), and (d) for validating Theorem 1 with respect to $a$, $\lambda$, $d_{s}$, and $v$, respectively. SM stands for the slap method. RW stands for the random walk. Model means the model prediction of the EST. Meas. means the measured mean searching time.

independent trials. At the beginning of each trial, we reset the robot position to be at $(0,0)$ and generate the target location according to a $2 \mathrm{D}$ uniform distribution. We then run the robot according to the selected motion plan and finish the trial as soon as the target is found.

\section{A. Validating Theorem 1 and Corollary 1}

We test Theorem 1 using both the slap method and the random walk because Theorem 1 is supposed to be independent of motion plans. The simulation is set up with different $a, \lambda$, $d_{s}$, and $v$ settings in Table I. In each setting, we collect both the model predicted EST and the measured mean searching time. The measured mean searching time is the average of the searching time over the $10 \mathrm{k}$ trials (the "Meas." values in Fig. 5). The model predicted ESTs, which are the "Model" values in Fig. 5, refer to the predicted ESTs according to the measured $D, \lambda, \tau_{\mathrm{IN}}$, and $\tau_{\text {OUT }}$ values in the experiment. In other words, we record their values and average them over the $10 \mathrm{k}$ trials and to obtain the estimation of $1 / \lambda, E(D)$, and $E\left(\tau_{\text {OUT }} \frac{e^{-\lambda \tau_{\mathrm{IN}}}}{1-e^{-\lambda \tau_{\mathrm{IN}}}}\right)$. We then feed them into $(6)$ to obtain the model prediction of the EST.

As illustrated in Fig. 5, the model prediction is fairly consistent with the measured mean searching time under all settings. There are more fluctuations between the model prediction and the measured mean searching time in random walk-based results than that of the slap method. This is expected because of more random factors associated with the random walk. Under the same trial number, the results from the random walk should contain more randomness.

\begin{tabular}{|c|c|c|c|c|}
\hline Figure & $a(\mathrm{~m})$ & $\lambda(1 / \mathrm{sec})$. & $d_{s}(\mathrm{~m})$ & $v(\mathrm{~m} / \mathrm{s})$ \\
\hline Fig. 5(a) & $100-1000$ & 0.1 & 1.0 & 1.0 \\
Fig. 5(b) & 200 & $0.1-1.0$ & 1.0 & 1.0 \\
Fig. 5(c) & 200 & 0.1 & $1-10$ & 1.0 \\
Fig. 5(d) & 200 & 0.1 & 1.0 & $0.01-100$ \\
Fig. 6 & 200 & 0.1 & 1.0 & 1.0 \\
\hline
\end{tabular}

TABLE I

PARAMETER SETTINGS FOR RESUlTS IN FIG. 5 AND FIg. 6.

The curve trends with respect to $a, \lambda, d_{s}$ and $v$ in Fig. 5 are also consistent with our analysis in (13) and (30). The EST increases as the field side length $a$ increases. The EST decreases as $\lambda, d_{s}$, and $v$ increase. All figures show that the random walk is slower than the slap method. In particular, Fig. 5 (a) is consistent with the asymptotical difference in Corollary 1.

\section{B. Validating Corollary 2}

We have also implemented both LCRT and ASER robot configurations. Again, the parameter settings are in the last row of Table I. The measured ESTs for both the configurations are shown in Fig. 6. It is clear that the EST for the LCRT is always much smaller than that of the ASER. This is consistent with Corollary 2. Curves in the figure also show the trend that the EST decreases as the $n$ increases. This is consistent with our analysis. Also, as $n$ gets very big, the curve levels at a non-zero value. This indicates that the signal transmission rate dominates the searching time. On the other hand, it is not desirable to arbitrarily increase $n$ because the marginal benefit 
would decrease.

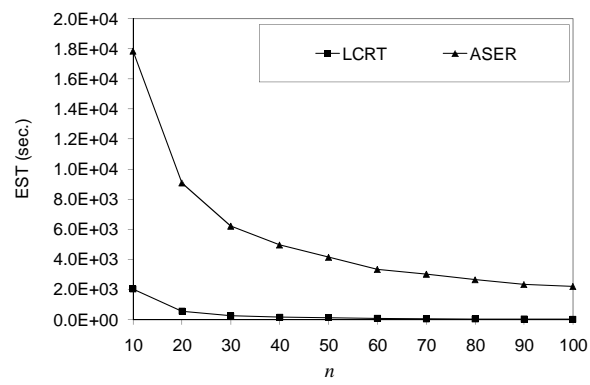

Fig. 6. Simulation results for comparing two robot configurations

\section{CONCLUSIONS AND FUTURE WORK}

We analytically modeled the expected searching time for a robot with a limited sensing range to search for a target that intermittently emits short duration signals. We presented the closed-form model for the EST. The EST model is motionplan independent and can be used to analyze different motion plans or robot configurations in two case studies. In the first case, we analyzed the slap method and the random walk and found that the slap method is asymptotically faster than the random walk. In the second case, the EST model revealed the interesting result that a low-cost robot team is always asymptotically faster than an expensive robot when the sensory coverage is the same. In both cases, the results demonstrated the usefulness and the capability of our EST analysis. Our theoretically results were extensively tested using simulation. The simulation results were consistent with the model.

This work will lead to a rich set of exciting future work. As an extension, we can analyze cases where multiple targets are needed to be searched. We can also develop the EST metrics for the searching of a moving target, an un-cooperating target, or multiple sensor combinations. Different sensor models can also be considered. Also, the searching space may exist obstacles. Applications of these extended results will be wideranging.

\section{ACKNOWLEDGEMENT}

Thanks for E. Frew, J. Xiao, and R. Volz for their insightful discussions. Thanks for Y. Xu, J. Zhang, A. Aghamohammadi, and W. Li for their inputs and contributions to the Networked Robots Laboratory in Texas A\&M University.

\section{REFERENCES}

[1] E. Gelenbe, N. Schmajuk, J. Staddon, and J. Reif, "Autonomous search by robots and animals: A survey," Robotics and Autonomous Systems, vol. 22, no. 1, pp. 23-34, 1997.

[2] H. Choset, "Coverage for robotics a survey of recent results," Annals of Mathematics and Artificial Intelligence, vol. 31, no. 1-4, pp. 113-126, Oct. 2001.

[3] S. Martinez, J. Cortes, and F. Bullo, "Motion coordination with distributed information," IEEE Control Systems Magazine, vol. 27, no. 4, pp. 75-88, August 2007.

[4] M. Schwager, D. Rus, and J. Slotine, "Decentralized, adaptive control for coverage with networked robots," International Journal of Robotics Research, vol. 28, no. 3, pp. 357-375, March 2009.

[5] M. Kao, J. Reif, and S. Tate, "Searching in an unknown environment: An optimal randomized algorithm for the cow-path problem," Information and Computation, vol. 131, no. 1, pp. 63-79, Nov. 1996.
[6] A. López-Ortiz and S. Schuierer, "Online parallel heuristics and robot searching under the competitive framework," in SWAT '02: Proceedings of the 8th Scandinavian Workshop on Algorithm Theory, 2002, pp. 260269.

[7] R. Baeza-Yates, J. Culberson, and J. Rawlins, "Searching in the plane," Information and Computation, vol. 106, no. 2, pp. 234-252, Oct. 1993.

[8] R. Motwani and R. Raghavan, Eds., Randomized Algorithms. Cambridge Univeristy Press, 1995.

[9] S. Thrun, W. Burgard, and D. Fox, Probabilistic Robotics. MIT Press, 2005.

[10] K. Laventall and J. Cortes, "Coverage control by multi-robot networks with limited-range anisotropic sensory," International Journal of Control, vol. 82, no. 6, pp. 1113 - 1121, June 2009.

[11] P. Brass, "Bounds on coverage and target detection capabilities for models of networks of mobile sensors," ACM Transactions on Sensor Networks (TOSN), vol. 3, no. 2, p. 9, June 2007.

[12] W. Burgard, M. Moors, D. Fox, R. Simmons, and S. Thrun, "Collaborative multi-robot exploration," in IEEE International Conference on Robotics and Automation, San Francisco, CA, Apr. 2000, pp. 476-481.

[13] M. Batalin and G. Sukhatme, "The design and analysis of an efficient local algorithm for coverage and exploration based on sensor network deployment," IEEE Transactions on Robotics, vol. 23, no. 4, pp. 661675, August 2007.

[14] W. Burgard, M. Moors, C. Stachniss, and F. Schneider, "Collaborative multi-robot exploration," IEEE Transactions on Robotics, vol. 21, no. 3, pp. 376-386, June 2005.

[15] D. Fox, J. Ko, K. Konolige, B. Limketkai, D. Schulz, and B. Stewart, "Distributed multirobot exploration and mapping," Proceedings of The IEEE, vol. 94, no. 7, pp. 1325-1339, July 2006.

[16] D. Song, J. Yi, and Z. Goodwin, "Localization of unknown networked radio sources using a mobile robot with a directional antenna," in the American Control Conference (ACC), New York City, USA, July, 2007, pp. 5952-5957.

[17] D. Song, C. Kim, and J. Yi, "Simultaneous localization of multiple unknown CSMA-based wireless sensor network nodes using a mobile robot with a directional antenna," Journal of Intelligent Service Robots, vol. 2, no. 4, pp. 219-233, Oct. 2009.

[18] _ - "Monte carlo simultaneous localization of multiple unknown transient radio sources using a mobile robot with a directional antenna," in IEEE International Conference on Robotics and Automation (ICRA), Kobe, Japan, May 2009.

[19] S. Ross, Introduction to Probability Models, Ninth Edition. Academic Press, 2007.

[20] F. Preparata and M. Shamos, Computational Geometry: An Introduction. Springer-Verlag, Berlin, 1985.

[21] J. Latombe, Robot Motion Planning. Kluwer Acadmic, Boston, MA, 1991.

[22] M. Brummelhuis and H. J. Hilhorst, "How a random walk covers a finite lattice," Physica A: Statistical Mechanics and its Applications, vol. 185, no. 1-4, pp. 35-44, June 1992.

[23] S. Condamin, O. Bénichou, and M. Moreau, "First-passage times for random walks in bounded domains," Physical Review Letters, vol. 95, no. 26, p. 260601, Dec 2005

[24] J. D. Noh and H. Rieger, "Random walks on complex networks," Physical Review Letters, vol. 92, no. 11, p. 118701, Mar. 2004.

[25] S. Condamin, O. Benichou, V. Tejedor, R. Voituriez, and J. Klafter, "First-passage times in complex scale-invarant media," Nature, vol. 450, pp. 77-80, Nov. 2007. 